\title{
Epitaxial niobium nitride superconducting nanowire single-photon detectors
}

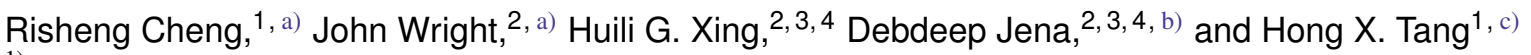 \\ 1) Department of Electrical Engineering, Yale University, New Haven, CT 06511, USA \\ ${ }^{2)}$ Department of Materials Science and Engineering, Cornell University, Ithaca, New York 14853, \\ USA. \\ ${ }^{3)}$ School of Electrical and Computer Engineering, Cornell University, Ithaca, New York 14853, \\ USA. \\ 4) Kavli Institute for Nanoscale Science, Cornell University, Ithaca, New York 14853, USA.
}

(Dated: 18 June 2020)

Superconducting nanowires used in single-photon detectors have been realized on amorphous or poly-crystalline films. Here, we report the use of single-crystalline $\mathrm{NbN}$ thin films for superconducting nanowire single-photon detectors (SNSPDs). Grown by molecular beam epitaxy (MBE) at high temperature on nearly lattice-matched AlN-on-sapphire substrates, the $\mathrm{NbN}$ films exhibit high degree of uniformity and homogeneity. Even with relatively thick films, the fabricated nanowire detectors show saturated internal efficiency at near-IR wavelengths, demonstrating the potential of MBE-grown NbN for realizing large arrays of on-chip SNSPDs and their integration with AlN-based $\chi^{(2)}$ quantum photonic circuits.

Superconducting nanowire single-photon detectors (SNSPDs) $)^{1,2}$ have become an indispensable resource for a range of quantum and classical applications due to their high detection efficiency over a broad spectrum ${ }^{3-6}$, ultra-fast speed $^{7,8}$, exceptional timing performance ${ }^{9-11}$, and ultra-low dark count noise $\mathrm{e}^{12,13}$. Two categories of superconducting materials have so far been used for the fabrication of highefficiency SNSPDs - poly-crystalline nitride, and amorphous alloy superconductors. SNSPDs patterned with thin-film amorphous superconducting materials, such as $\mathrm{WSi}^{3,14}$ and $\mathrm{MoSi}^{15-17}$, have exhibited excellent homogeneity over a large device area ${ }^{18-20}$ due to the absence of grain boundaries. However, they require relatively lower operation temperature and have lower maximum counting rates, resulting from longer hot spot relaxation time in comparison with the SNSPDs made from nitride superconductors, such as $\mathrm{NbN}^{21-24}$ and $\mathrm{NbTiN}^{25-29}$. On the other hand, $\mathrm{Nb}(\mathrm{Ti}) \mathrm{N}$-based detectors have shown relatively superior timing performance, demonstrating $<3$ ps jitter measured with a short straight nanowire ${ }^{10}$ and $<8$ ps with a large-area meandered nanowires ${ }^{30}$. Despite these advantages, the homogeneity of $\mathrm{Nb}(\mathrm{Ti}) \mathrm{N}-\mathrm{SNSPD}$ are ultimately limited by the poly-crystalline nature of the sputtered $\mathrm{Nb}(\mathrm{Ti}) \mathrm{N}$ films, which could lead to non-uniform distribution of critical currents in a large array of singlephoton detectors required for future integrated quantum photonic circuits.

In this Letter, we demonstrate SNSPDs made from singlecrystal $\mathrm{NbN}$ thin films grown by molecular beam epitaxy $(\mathrm{MBE})^{31}$ on nearly lattice-matched AlN-on-sapphire substrates. This substrate platform is attractive for the integration of SNSPDs with several other elements of nitride-based photonic integrated circuits ${ }^{32-36}$. The epitaxial $\mathrm{NbN}$ films exhibit a high degree of thickness uniformity and structural perfection

\footnotetext{
a) These authors contributed equally to this work.

b) Electronic mail: djena@cornell.edu

c)Electronic mail: hong.tang@yale.edu
}

(a)

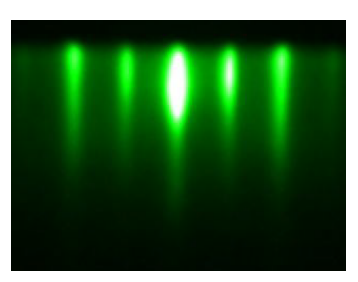

(c)

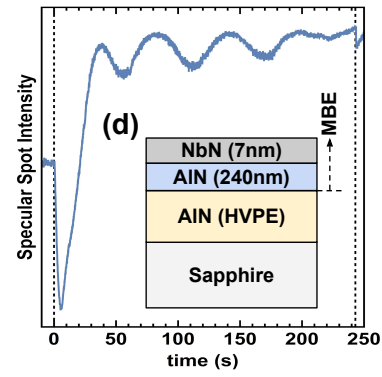

(e)

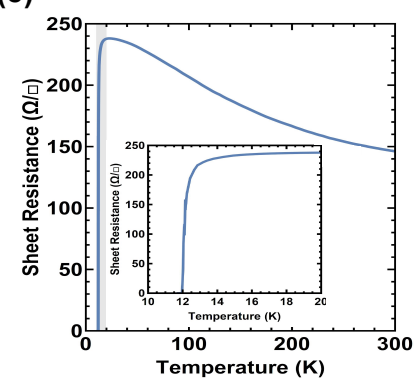

FIG. 1 : (a) In-situ RHEED pattern measured after the film growth demonstrating the epitaxial nature of the $\mathrm{NbN}$ film. The streakiness of the pattern evidences that the surface is effectively 2D. (b) AFM surface height map of the $\mathrm{NbN}$ thin film exhibiting $R_{\mathrm{rms}}=0.29 \mathrm{~nm}$. (c) RHEED intensity monitored throughout the $\mathrm{NbN}$ thin film growth. The exhibited oscillations of the specular spot brightness indicates the 2D layer-by-layer growth mode of the $\mathrm{NbN}$. (d) Crosssectional sketch of the thin film layer structure. (e) Measured sheet resistance of the $\mathrm{NbN}$ thin film versus temperature with the inset showing the $T_{\mathrm{c}}$ of $12.1 \mathrm{~K}$.

owing to the 2D layer-by-layer growth unique to MBE technique. The fabricated device consisting of $20 \mathrm{~nm}$-wide and $7 \mathrm{~nm}$-thick nanowire shows saturated internal efficiency at the wavelength of $780 \mathrm{~nm}$ and $1050 \mathrm{~nm}$, while further reduction in achievable thin film thickness holds promise for saturating the efficiency at longer wavelength with more relaxed wire width. We expect MBE-NbN on AlN-on-sapphire substrate 
(a)

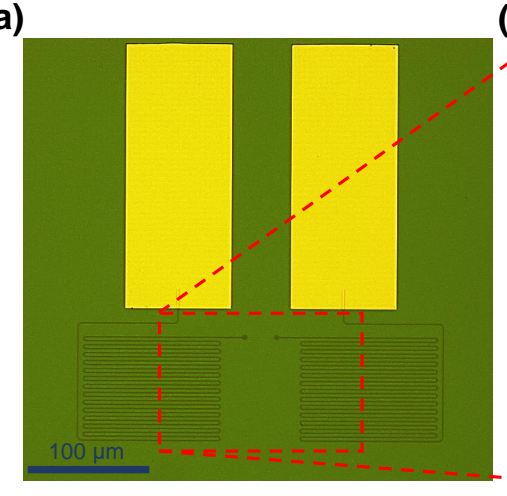

(b)

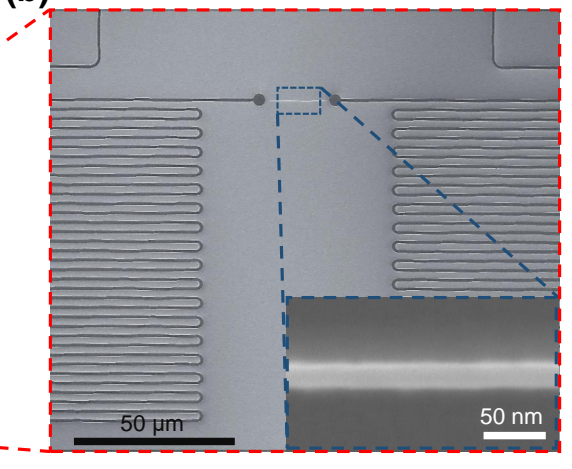

(c)

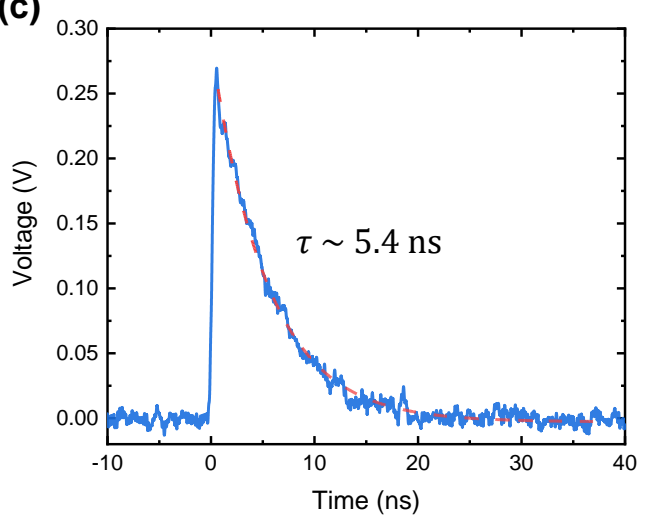

FIG. 2 : (a) Optical micrograph image of the fabricated SNSPD device. (b) Close-up scanning electron micrograph (SEM) image of the active straight nanowire and series inductor made of $1 \mu \mathrm{m}$-wide meandered wire. The inset shows the further zoom-in view of the $20 \mathrm{~nm}$-wide straight nanowire. (c) Single-shot trace of output voltage pulse from the $20 \mathrm{~nm}$-wide detector measured by a $4 \mathrm{GHz}$ oscilloscope. The decay time constant $(\tau)$ extracted from the exponential fitting (red dashed line) is $5.4 \mathrm{~ns}$.

shown here could provide a scalable material platform for realizing large array of on-chip SNSPDs and integration with nitride-based photonic circuits.

As illustrated in Fig. 1, epitaxial $\mathrm{NbN}$ films are grown by radio-frequency plasma-assisted MBE on a commercial 2 inch-diameter c-plane sapphire substrate with a $3 \mu \mathrm{m}$-thick AlN film grown by hydride vapor phase epitaxy (HVPE). A $240 \mathrm{~nm}$-thick AlN film of Al-polar orientation is grown by MBE, followed by the growth of $\mathrm{NbN}$ as shown in Fig. 1(d). During the growth of the films, the reactive nitrogen is generated using a radio-frequency plasma source fed by ultrahighpurity $\mathrm{N}_{2}$ gas, which is further purified by an in-line purifier. Aluminum (99.9999\% purity) is supplied using a Knudsen effusion cell. The $\mathrm{Nb}$ flux is generated using an in situ electronbeam evaporator source with 3N5-pure (excluding tantalum, $\mathrm{Ta}) \mathrm{Nb}$ pellets in a tungsten hearth liner. The $\mathrm{NbN}$ films are grown at the temperature of $1100{ }^{\circ} \mathrm{C}$ measured by a thermocouple behind the substrate, at a growth rate of approximately $1.0 \mathrm{~nm} / \mathrm{min}$.

The MBE film growth is monitored in situ using a reflection high-energy electron diffraction (RHEED) system operated at $15 \mathrm{kV}$ voltage and $1.5 \mathrm{~A}$ current. Figure 1(a) shows sharp and streaky patterns formed by electron diffraction from the smooth surface of the $\mathrm{NbN}$ film, indicating the epitaxial nature of the single-crystal NbN film. As shown in Fig. 1(c), the in situ observation of oscillations of the RHEED intensity versus the growth time confirms that the $\mathrm{NbN}$ grows in a $2 \mathrm{D}$ layer-by-layer growth mode on the AlN surface. The thickness of the $\mathrm{NbN}$ film is $7.0 \mathrm{~nm}$, measured by X-ray reflectivity (XRR) with a Rigaku SmartLab diffractometer using $\mathrm{CuK} \alpha 1$ radiation. Figure 1(b) shows the morphology of the $\mathrm{NbN}$ film surface characterized employing tapping mode atomic force microscopy (AFM); the root-mean-square roughness $\left(R_{\mathrm{rms}}\right)$ of the film surface is less than $0.3 \mathrm{~nm}$ within a scan size of $1 \mu \mathrm{m} \times 1 \mu \mathrm{m}$. In addition, the crystal orientation of the $\mathrm{NbN}$ is determined using RHEED and X-ray diffraction (XRD), which indicates the cubic $\mathrm{NbN}$ grows with the $\left\{\begin{array}{lll}1 & 1 & 1\end{array}\right\}$ crystal axis aligned to the c-axis of AlN.
Figure 1(e) shows the temperature dependence of the sheet resistance of the MBE-NbN thin film with the inset showing a zoom-in view of the superconducting transition region. The transition temperature of the film is measured to be $T_{\mathrm{c}}=12.1 \mathrm{~K}$, defined as the temperature where the resistance of the film drops to $50 \%$ of the normal-state resistance measured at $20 \mathrm{~K}$. This value is among one of the highest results reported so far for $\mathrm{NbN}$ thin films of a few nanometer thickness. The high $T_{\mathrm{c}}$ value is also consistent with the significantly low resistivity of the film, which is calculated to be only $\sim 100 \mu \Omega \cdot \mathrm{cm}$ obtained by multiplying the thickness with the room-temperature sheet resistance.

We fabricate SNSPD devices by patterning the $7 \mathrm{~nm}$-thick MBE-NbN film. The nanowires are defined by the exposure of negative-tone $6 \%$ hydrogen silsesquioxane (HSQ) resist using $100 \mathrm{kV}$ electron-beam lithography (Raith EBPG 5200) and the subsequent development in $25 \%$ tetramethylammonium hydroxide (TMAH) for 2 minutes at room temperature. The HSQ resist is spun at the speed of $4000 \mathrm{rpm}$, resulting in an approximate thickness of $90 \mathrm{~nm}$. In a second electron-beam lithography step, contact electrodes are defined using doublelayer polymethyl methacrylate (PMMA) positive-tone resist. After the development in the mixture of methyl isobutyl ketone (MIBK) and isopropyl alcohol (IPA), we liftoff electronbeam evaporated $10 \mathrm{~nm}$-thick $\mathrm{Cr}$ adhesion layer and $100 \mathrm{~nm}$ thick $\mathrm{Au}$ in acetone overnight to form the contact pads. The HSQ nanowire pattern is then transferred to the $\mathrm{NbN}$ layer in a timed reactive-ion etching (RIE) step employing $\mathrm{CF}_{4}$ chemistry and $50 \mathrm{~W}$ RF power. The HSQ resist is left on top of the $\mathrm{NbN}$ nanowires after fabrication, serving as a barrier to oxidation.

For initial tests, we fabricate short-nanowire detectors with widths ranging from $20 \mathrm{~nm}$ to $100 \mathrm{~nm}$ for comparison of the internal efficiencies. As shown in Fig. 2(a) and (b), the active detection parts of the devices are made of $20 \mu \mathrm{m}$-long straight nanowires which are suitable for future waveguide integration. All the nanowires are serially connected to long $1 \mu \mathrm{m}$-wide meandered wires to prevent the detector latching at high bias 


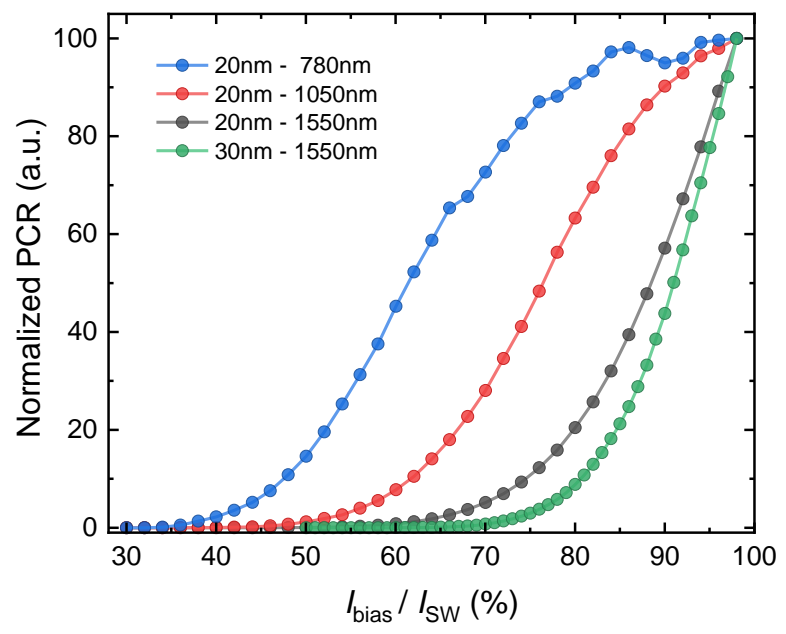

FIG. 3 : Normalized photon counting rates (PCR) versus the relative bias current $\left(I_{\text {bias }} / I_{\mathrm{SW}}\right)$ measured with the $20 \mathrm{~nm}$-wide and $30 \mathrm{~nm}$ wide nanowire detectors for varying wavelength of photons. $I_{\mathrm{SW}}$ of the nanowires are measured to be $25.5 \mu \mathrm{A}$ and $38.8 \mu \mathrm{A}$, respectively.

currents. The sheet resistance of the devices are measured to be around $180 \Omega / \mathrm{sq}$, which slightly increase compared to the value measured on the bare film prior to fabrication.

In order to characterize the optical response of the fabricated detectors, the detector chip containing multiple devices is mounted on a 3-axis stack of Attocube stages inside a closed-cycle refrigerator and cooled down to $1.7 \mathrm{~K}$ base temperature. Continuous wave $(\mathrm{CW})$ laser light with varied wavelength is attenuated to the single-photon level and sent to the detector chip via a standard telecommunication fiber (SMF-28) installed in the refrigerator. The detectors are floodilluminated by fixing the fiber tip far away from the surface of the detector chip. We control the Attocube stages to move the detector chip at low temperature and make the electrical contact between the RF probes and the gold pads of the detectors. The RF probes are connected to a semi-rigid coaxial cable installed in the refrigerator, while the room-temperature end of the cable is attached to a bias-tee (Mini-Circuits ZFBT$6 \mathrm{GW}+$ ) to separate the DC bias current and RF output pulses for the detectors. The bias current is supplied by a programmable sourcemeter (Keithley 2401) in conjunction with a low-pass filter ( $1 \mathrm{kHz}$ cut-off frequency). The output pulses of the detectors are amplified by a low-noise RF amplifier (RF bay LNA-650) and sent to a $4 \mathrm{GHz}$ oscilloscope for the pulse observation or a pulse counter (PicoQuant PicoHarp 300) for the photon counting measurement. Figure 2(c) shows a singleshot trace measurement of the output voltage pulse from the $20 \mathrm{~nm}$-wide detector. The decay time constant extracted from the exponential fitting (red dashed line) is $5.4 \mathrm{~ns}$, which translates into $24 \mathrm{pH} / \mathrm{sq}$ sheet kinetic inductance of the $\mathrm{NbN}$ film, assuming $50 \Omega$ input impedance of the readout amplifier.

Figure 3 demonstrates the normalized photon counting rates (PCR) as a function of the relative bias current to the switching current $\left(I_{\text {bias }} / I_{\text {SW }}\right)$ for $20 \mathrm{~nm}$-wide and $30 \mathrm{~nm}$-wide nanowire detectors. $I_{\mathrm{SW}}$ of the devices are measured to be
$25.5 \mu \mathrm{A}$ and $38.8 \mu \mathrm{A}$, respectively. As expected, detectors made of narrower nanowires with reduced $I_{\mathrm{SW}}$ show better saturated internal efficiencies at shorter wavelength. For $20 \mathrm{~nm}$ wide nanowire detector, we observe a broad saturation plateau at $780 \mathrm{~nm}$ wavelength, while the efficiency is only nearly saturated at $1050 \mathrm{~nm}$ wavelength. The minor fluctuation in the curve corresponding to $780 \mathrm{~nm}$ wavelength is due to the polarization instability of the laser, since the photon absorption of the nanowire is significantly dependent on the polarization status of the incident photons. Neither the $20 \mathrm{~nm}$ wide nor the $30 \mathrm{~nm}$-wide nanowires show saturation behavior at $1550 \mathrm{~nm}$ wavelength. We attribute the inefficiency of the detectors to the significantly low electrical resistivity of the MBE-NbN material. In comparison with the SNSPDs made from sputtered ${ }^{21}$ or atomic-layer-deposited (ALD) ${ }^{24,37} \mathrm{NbN}$ film of comparable thickness, the nanowires shown in this work demonstrate approximately 3 times reduced sheet resistance as well as sheet kinetic inductance, and in the meantime, 3-5 times improved critical current density. All of these results are in agreement with the 2-3 times lower resistivity of MBE-NbN compared to sputtered or ALD-NbN. Thus, we expect that by further reducing the MBE NbN film thickness down to 2-3 nm, saturated efficiency can be obtained in longer wavelengths with relaxed nanowire widths up to $100 \mathrm{~nm}$. The growth of high crystalline quality $\mathrm{NbN}$ films of $3 \mathrm{~nm}$ thick or less are achievable by MBE, although a method to protect such thin films from oxidation upon exposure to the ambient is necessary and under investigation. Future work will explore the suitability of such ultra-thin films for SNSPDs.

In summary, we have demonstrated the first SNSPDs patterned from MBE-grown single-crystal $\mathrm{NbN}$ thin films on AlN. The $20 \mathrm{~nm}$-wide SNSPDs show saturated internal efficiency at the wavelength of $780 \mathrm{~nm}$ and $1050 \mathrm{~nm}$. It is worth mentioning that the AlN-on-sapphire substrate, which the epitaxial growth of $\mathrm{NbN}$ relies on, is particularly attractive due to its potential of the on-chip integration of SNSPDs with versatile AIN nanophotonic circuits. The excellent optical functionalities of AlN, such as strong $\chi^{(2)} / \chi^{(3)}$ nonlinearity $^{38}$ and large electro-optic effect ${ }^{39,40}$, renders $\mathrm{NbN}$ on AlN-onsapphire a very attractive material platform for realizing fully integrated quantum photonic circuits with the generation, routing, active manipulation and the final detection of single photons on a single chip.

\section{ACKNOWLEDGMENTS}

This project is funded by Office of Naval Research grants (N00014-20-1-2126 and N00014-20-1-2176) monitored by Dr. Paul Maki. D.J. acknowledges funding support from NSF RAISE TAQs Award No. 1839196 monitored by Dr. D. Dagenais. H.X.T. acknowledges funding support from DARPA DETECT program through an ARO grant (No: W911NF-162-0151), NSF EFRI grant (EFMA-1640959) and the Packard Foundation. The authors would like to thank Sean Rinehart, Kelly Woods, Dr. Yong Sun, and Dr. Michael Rooks at Yale University for their assistance provided in the device fabrication, and Dr. Scott Katzer and Dr. David Meyer at the Naval Research Laboratory for useful discussions. The epitaxial growth was performed at Cornell University, and ma- 
terial characterization used resources made available by the NSF CCMR MRSEC Award No. 1719875. The fabrication of the devices was done at the Yale School of Engineering \& Applied Science (SEAS) Cleanroom and the Yale Institute for Nanoscience and Quantum Engineering (YINQE).

\section{REFERENCES}

${ }^{1}$ G. Gol'Tsman, O. Okunev, G. Chulkova, A. Lipatov, A. Semenov, K. Smirnov, B. Voronov, A. Dzardanov, C. Williams, and R. Sobolewski, "Picosecond superconducting single-photon optical detector," Applied Physics Letters 79, 705 (2001).

${ }^{2}$ R. H. Hadfield, "Single-photon detectors for optical quantum information applications," Nature Photonics 3, 696 (2009).

${ }^{3}$ F. Marsili, V. B. Verma, J. A. Stern, S. Harrington, A. E. Lita, T. Gerrits, I. Vayshenker, B. Baek, M. D. Shaw, R. P. Mirin, and S. W. Nam, "Detecting single infrared photons with $93 \%$ system efficiency," Nature Photonics 7, 210 (2013).

${ }^{4}$ V. Verma, A. Lita, B. Korzh, E. Wollman, M. Shaw, R. Mirin, and S. Nam, "Towards single-photon spectroscopy in the mid-infrared using superconducting nanowire single-photon detectors," in Advanced Photon Counting Techniques XIII, Vol. 10978 (International Society for Optics and Photonics, 2019) p. 109780N.

${ }^{5}$ F. Marsili, F. Bellei, F. Najafi, A. Dane, E. Dauler, R. J. Molnar, and K. K. Berggren, "Efficient single photon detection from $500 \mathrm{~nm}$ to $5 \mu \mathrm{m}$ wavelength," Nano Letters 12, 4799 (2012).

${ }^{6}$ D. V. Reddy, A. E. Lita, S. W. Nam, R. P. Mirin, and V. B. Verma, "Achieving $98 \%$ system efficiency at $1550 \mathrm{~nm}$ in superconducting nanowire single photon detectors," in Rochester Conference on Coherence and Quantum Optics (CQO-11) (OSA, 2019).

${ }^{7}$ J. Münzberg, A. Vetter, F. Beutel, W. Hartmann, S. Ferrari, W. H. Pernice, and C. Rockstuhl, "Superconducting nanowire single-photon detector implemented in a 2d photonic crystal cavity," Optica 5, 658 (2018).

${ }^{8}$ W. Zhang, J. Huang, C. Zhang, L. You, C. Lv, L. Zhang, H. Li, Z. Wang, and X. Xie, "A 16-pixel interleaved superconducting nanowire single-photon detector array with a maximum count rate exceeding $1.5 \mathrm{GHz}$," IEEE Transactions on Applied Superconductivity 29, 1 (2019).

${ }^{9}$ I. E. Zadeh, J. W. Los, R. Gourgues, G. Bulgarini, S. M. Dobrovolskiy, V. Zwiller, and S. N. Dorenbos, "A single-photon detector with high efficiency and sub-10ps time resolution," arXiv preprint arXiv:1801.06574 (2018).

${ }^{10}$ B. Korzh, Q.-Y. Zhao, J. P. Allmaras, S. Frasca, T. M. Autry, E. A. Bersin, A. D. Beyer, R. M. Briggs, B. Bumble, M. Colangelo, G. M. Crouch, A. E. Dane, T. Gerrits, A. E. Lita, F. Marsili, G. Moody, C. Peña, E. Ramirez, J. D. Rezac, N. Sinclair, M. J. Stevens, A. E. Velasco, V. B. Verma, E. E. Wollman, S. Xie, D. Zhu, P. D. Hale, M. Spiropulu, K. L. Silverman, R. P. Mirin, S. W. Nam, A. G. Kozorezov, M. D. Shaw, and K. K. Berggren, "Demonstration of sub-3 ps temporal resolution with a superconducting nanowire single-photon detector," Nature Photonics (2020).

${ }^{11}$ I. E. Zadeh, J. W. Los, R. Gourgues, J. Chang, A. W. Elshaari, J. Zichi, Y. J. van Staaden, J. Swens, N. Kalhor, A. Guardiani, et al., "A platform for high performance photon correlation measurements," arXiv preprint arXiv:2003.09916 (2020).

${ }^{12}$ C. Schuck, W. H. P. Pernice, and H. X. Tang, "Waveguide integrated low noise NbTiN nanowire single-photon detectors with milli-Hz dark count rate," Scientific Reports 3, 1893 (2013).

${ }^{13}$ H. Shibata, K. Shimizu, H. Takesue, and Y. Tokura, "Ultimate low system dark-count rate for superconducting nanowire single-photon detector," $\mathrm{Op}$ tics Letters 40, 3428 (2015).

${ }^{14}$ B. Baek, A. E. Lita, V. Verma, and S. W. Nam, "Superconducting a$\mathrm{W}_{x} \mathrm{Si}_{1-x}$ nanowire single-photon detector with saturated internal quantum efficiency from visible to $1850 \mathrm{~nm}$," Applied Physics Letters 98, 251105 (2011).

${ }^{15}$ M. Caloz, M. Perrenoud, C. Autebert, B. Korzh, M. Weiss, C. Schönenberger, R. J. Warburton, H. Zbinden, and F. Bussières, "High-detection efficiency and low-timing jitter with amorphous superconducting nanowire single-photon detectors," Applied Physics Letters 112, 061103 (2018).

${ }^{16}$ Y. P. Korneeva, M. Y. Mikhailov, Y. P. Pershin, N. Manova, A. Divochiy, Y. B. Vakhtomin, A. Korneev, K. Smirnov, A. Sivakov, A. Y. Devizenko, et al., "Superconducting single-photon detector made of MoSi film," Superconductor Science and Technology 27, 095012 (2014).

${ }^{17}$ J. Li, R. A. Kirkwood, L. J. Baker, D. Bosworth, K. Erotokritou, A. Banerjee, R. M. Heath, C. M. Natarajan, Z. H. Barber, M. Sorel, et al., "Nanooptical single-photon response mapping of waveguide integrated molybdenum silicide (MoSi) superconducting nanowires," Optics Express 24, 13931 (2016).

${ }^{18}$ J. Chiles, S. M. Buckley, A. Lita, V. B. Verma, J. Allmaras, B. Korzh, M. D. Shaw, J. M. Shainline, R. P. Mirin, and S. W. Nam, "Superconducting microwire detectors with single-photon sensitivity in the near-infrared," arXiv preprint arXiv:2002.12858 (2020).

${ }^{19}$ I. Charaev, Y. Morimoto, A. Dane, A. Agarwal, M. Colangelo, and K. K. Berggren, "Large-area microwire MoSi single-photon detectors at $1550 \mathrm{~nm}$ wavelength," Applied Physics Letters 116, 242603 (2020).

${ }^{20}$ E. E. Wollman, V. B. Verma, A. E. Lita, W. H. Farr, M. D. Shaw, R. P. Mirin, and S. W. Nam, "Kilopixel array of superconducting nanowire singlephoton detectors," Optics Express 27, 35279 (2019).

${ }^{21}$ W. Zhang, L. You, H. Li, J. Huang, C. Lv, L. Zhang, X. Liu, J. Wu, Z. Wang, and $\mathrm{X}$. Xie, "NbN superconducting nanowire single photon detector with efficiency over $90 \%$ at $1550 \mathrm{~nm}$ wavelength operational at compact cryocooler temperature," Science China Physics, Mechanics \& Astronomy 60, 120314 (2017).

${ }^{22}$ O. Kahl, S. Ferrari, V. Kovalyuk, G. N. Goltsman, A. Korneev, and W. H. Pernice, "Waveguide integrated superconducting single-photon detectors with high internal quantum efficiency at telecom wavelengths," Scientific Reports 5, 10941 (2015).

${ }^{23}$ W. Pernice, C. Schuck, O. Minaeva, M. Li, G. Goltsman, A. Sergienko, and H. Tang, "High-speed and high-efficiency travelling wave single-photon detectors embedded in nanophotonic circuits," Nature Communications $\mathbf{3}$, 1325 (2012).

${ }^{24}$ R. Cheng, S. Wang, and H. X. Tang, "Superconducting nanowire singlephoton detectors fabricated from atomic-layer-deposited NbN," Applied Physics Letters 115, 241101 (2019).

${ }^{25}$ I. Esmaeil Zadeh, J. W. Los, R. B. Gourgues, V. Steinmetz, G. Bulgarini, S. M. Dobrovolskiy, V. Zwiller, and S. N. Dorenbos, "Single-photon detectors combining high efficiency, high detection rates, and ultra-high timing resolution," APL Photonics 2, 111301 (2017).

${ }^{26}$ R. Cheng, M. Poot, X. Guo, L. Fan, and H. X. Tang, "Large-area superconducting nanowire single-photon detector with double-stage avalanche structure," IEEE Transactions on Applied Superconductivity 27, 1 (2017).

${ }^{27}$ R. Cheng, X. Guo, X. Ma, L. Fan, K. Y. Fong, M. Poot, and H. X. Tang, "Self-aligned multi-channel superconducting nanowire single-photon detectors," Optics Express 24, 27070 (2016).

${ }^{28}$ S. Miki, M. Yabuno, T. Yamashita, and H. Terai, "Stable, high-performance operation of a fiber-coupled superconducting nanowire avalanche photon detector," Optics Express 25, 6796 (2017).

${ }^{29}$ H. Machhadani, J. Zichi, C. Bougerol, S. Lequien, J.-L. Thomassin, N. Mollard, A. Mukhtarova, V. Zwiller, J.-M. Gérard, and E. Monroy, "Improvement of the critical temperature of NbTiN films on III-nitride substrates," Superconductor Science and Technology 32, 035008 (2019).

${ }^{30}$ I. E. Zadeh, J. W. N. Los, R. B. M. Gourgues, J. Chang, A. W. Elshaari, J. R. Zichi, Y. J. van Staaden, J. P. E. Swens, N. Kalhor, A. Guardiani, Y. Meng, K. Zou, S. Dobrovolskiy, A. W. Fognini, D. R. Schaart, D. Dalacu, P. J. Poole, M. E. Reimer, X. Hu, S. F. Pereira, V. Zwiller, and S. N. Dorenbos, "Efficient single-photon detection with 7.7 ps time resolution for photon correlation measurements," ACS Photonics (2020).

${ }^{31}$ R. Yan, G. Khalsa, S. Vishwanath, Y. Han, J. Wright, S. Rouvimov, D. S. Katzer, N. Nepal, B. P. Downey, D. A. Muller, H. G. Xing, D. J. Meyer, and D. Jena, "GaN/NbN epitaxial semiconductor/superconductor heterostructures," Nature 555, 183 (2018).

${ }^{32}$ S. Ferrari, C. Schuck, and W. Pernice, "Waveguide-integrated superconducting nanowire single-photon detectors," Nanophotonics 7 (2018).

${ }^{33}$ R. Cheng, C.-L. Zou, X. Guo, S. Wang, X. Han, and H. X. Tang, "Broadband on-chip single-photon spectrometer," Nature Communications 10, 1 (2019).

${ }^{34}$ A. Gaggero, F. Martini, F. Mattioli, F. Chiarello, R. Cernansky, A. Politi, and R. Leoni, "Amplitude-multiplexed readout of single photon detectors based on superconducting nanowires," Optica 6, 823 (2019).

${ }^{35}$ F. Najafi, J. Mower, N. C. Harris, F. Bellei, A. Dane, C. Lee, X. Hu, P. Kharel, F. Marsili, S. Assefa, K. K. Berggren, and D. Englund, "On-chip 
detection of non-classical light by scalable integration of single-photon detectors," Nature Communications 6, 5873 (2015).

${ }^{36}$ R. Cheng, S. Wang, C.-L. Zou, and H. Tang, "Design of micron-long superconducting nanowire perfect absorber for efficient high speed single-photon detection," Photonics Research (2020).

${ }^{37}$ A. A. Sayem, R. Cheng, S. Wang, and H. X. Tang, "Lithium-niobate-oninsulator waveguide-integrated superconducting nanowire single-photon detectors," arXiv preprint arXiv:1912.09418 (2019).
${ }^{38}$ X. Guo, C.-L. Zou, C. Schuck, H. Jung, R. Cheng, and H. X. Tang, "Parametric down-conversion photon-pair source on a nanophotonic chip," Light: Science \& Applications 6, e16249 (2017).

${ }^{39}$ L. Fan, C.-L. Zou, R. Cheng, X. Guo, X. Han, Z. Gong, S. Wang, and H. X. Tang, "Superconducting cavity electro-optics: A platform for coherent photon conversion between superconducting and photonic circuits," Science Advances 4, eaar4994 (2018).

${ }^{40}$ C. Xiong, W. H. P. Pernice, X. Sun, C. Schuck, K. Y. Fong, and H. X. Tang, "Aluminum nitride as a new material for chip-scale optomechanics and nonlinear optics," New Journal of Physics 14, 095014 (2012). 